\title{
All-optical measurements of elastic constants in nematic liquid crystals
}

\author{
Bartłomiej W. Klus, Waldemar K. Bajdecki and Mirosław A. Karpierz \\ Faculty of Physics, Warsaw University of Technology, Koszykowa 75, 00-662 Warszawa, \\ Received March 12, 2012; accepted March 23, 2012; published March 31, 2012
}

\begin{abstract}
Reorientation of liquid crystals induced by light is used as a method to measure elastic constants. Especially this all-optical method can determine constant connected with twist deformation, which is not easy in conventionally known methods. Experimental results show very good accuracy in comparison with other methods.
\end{abstract}

Nematic liquid crystals (NLCs) are very a important material in photonics due to their optical anisotropy easily reoriented by external fields [1]. The most important parameters of this structure are birefringence, electric anisotropy and Frank's elastic constants. Elastic constants are connected with the value of external electric field which is necessary to change orientation of molecules. Therefore measurements of these constants are very important for applications of NLCs. Elastic constants are measured usually in setups based on electro-optical effect. In these methods a slow-variable external electric field is used to reorient molecules in NLCs cell [2]. In this letter we present the measurement of Frank elastic constants in several NLCs by using an all-optical method based on a nonlinear self-diffraction effect where reorientation is included by an optical wave [3, 4].

The initial orientation of NLCs is induced at the boundaries of the NLC cell. In our application we used two configurations: planar (homogenic) texture (Fig.1.a) where anisotropic molecules are oriented parallel to the border between NLC and glass, and homeotropic texture (Fig.1.b) where molecules are perpendicular to this border. These orientations of NLC can be changed by the external electric or magnetic fields. The strength of reorientation can be described by three basic deformations: splay, twist and bend, which are connected with three Frank's elastic constants $\mathrm{K}_{11}$ (splay), $\mathrm{K}_{22}$ (twist) and $\mathrm{K}_{33}$ (bend). In typical NLCs the elastic constants have the value in the range $10^{-12} \div 10^{-11} \mathrm{~N}$.

The density of free energy in NLCs is equal to:

$f=\frac{1}{2} K_{11}(\nabla \cdot \vec{n})^{2}+\frac{1}{2} K_{22}(\vec{n} \cdot \nabla \times \vec{n})^{2}+\frac{1}{2} K_{33}(\vec{n} \times \nabla \times \vec{n})^{2}-\frac{\varepsilon_{0} \Delta \varepsilon}{2}(\vec{n} \cdot \vec{E})^{2}$

*E-mail: bartklus@if.pw.edu.pl where $\vec{n}$ is a director describing the mean direction of molecular alignment. The last term in Eq. (1) is connected with the energy of interaction between an electric field and anisotropic molecules (where $\Delta \varepsilon$ is an electrical anisotropy and $\varepsilon_{0}$ is the electric permittivity of the free space).

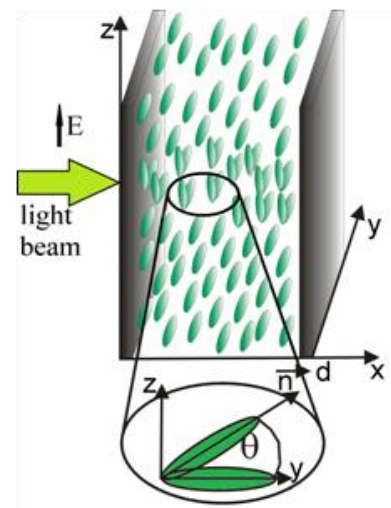

a)

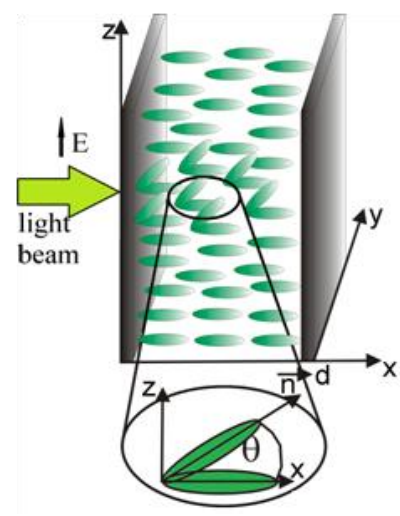

b)
Fig.1. Analyzed configuration of NLCs cells with a) planar, b) homeotropic textures.

The minimization of total energy $\iiint f d V$ can give the orientation of NLC. The minimization of the total energy leads to the Euler-Lagrange equations which, for homogeneous electric field in analyzed configurations, are reduced to the following form [5]:

$$
K_{i i} \frac{d^{2} \theta}{d x^{2}}+\frac{\varepsilon_{0} \Delta \varepsilon}{2} E^{2} \sin 2 \theta=0
$$

where $\theta$ is an angle between the director and the $y$-axis [in a planar configuration (Fig. 1.a)] or the $\mathrm{x}$-axis [homeotropic configuration (Fig. 1.b)]. In both textures the boundary conditions are $\theta(0)=\theta(d)=0$, where $d$ is the thickness of the cell. In a planar configuration the electric field induces twist deformation and therefore $K_{\mathrm{ii}}=K_{22}$ in Eq. (2), while in homeotropic configuration the most important is the bend deformation and $K_{\mathrm{ii}}=K_{33}$ [2].

In a case when the electric field is orthogonal to the director, the Freedericksz effect can be observed. In this phenomenon, to reorient molecules we have to use an 
electric field which is larger than the threshold value $E_{t h}$. This threshold value can be obtained from Eq. (2) in a case when $\sin \theta \approx \theta(\theta<1)$ and it is equal to:

$$
E_{t h}^{(i)}=\frac{\pi}{d} \sqrt{\frac{K_{i i}}{\varepsilon_{0} \Delta \varepsilon}}
$$

Reorientation is also induced by the electric field of electro-magnetic wave $E_{\mathrm{opt}}$. In such a case, the electric field in Eqs. (1-3) should be replaced by an average value $E^{2}=0.5 E_{o p t}^{2}$, and electrical anisotropy should be taken for the optical frequency $\Delta \varepsilon=n_{e}{ }^{2}-n_{o}{ }^{2}$, where $n_{o}$ and $n_{e}$ are ordinary and extraordinary refractive indices. For a light beam the Freedericksz effect appears when the electric field in the center of the beam is larger than the threshold value. For a Gaussian beam the beam power is equal [3-4]:

$$
P=\int_{0}^{\infty} \int_{0}^{2 \pi} I_{o} r d r d \varphi=\sqrt{\frac{\varepsilon \varepsilon_{0}}{\mu \mu_{0}}} \frac{\pi w_{0}^{2}}{4} n_{o} E_{o}{ }^{2}
$$

where: $\mathrm{I}_{\mathrm{o}}$ is an intensity and $E_{o}$ is an electric field in the beam center, $w_{0}$ is the beam waist and $\mu_{0}$ and $\mu$ are magnetic permeability of the free space and material. For diamagnetic materials $\mu \approx 1$ and thus the permittivity is equal to $\varepsilon=n_{o}^{2}$. Reorientation appears when the electrical field in the center of the beam is larger than threshold value $\left(E_{o} \geq E_{t h}\right)$, which means that the beam power is larger than corresponding to the threshold power $P_{t h}$. From measurements of this threshold power it is possible measure the elastic constants:

$$
K_{i i}=2 P_{t h} \frac{d^{2} \Delta \varepsilon}{\pi^{3} w_{0}^{2} n_{0}^{2} c}
$$

The experimental setup for Frank's elastic constants measurements are presented in Fig. 2. As a light source a Ti:sapphire laser with $\lambda=793 \mathrm{~nm}$ and an $\mathrm{Nd}$ :YAG laser with $\lambda=532 \mathrm{~nm}$ were used.

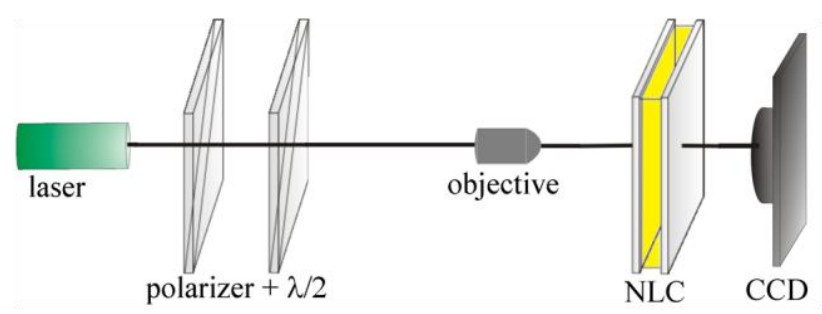

Fig. 2. Experimental setup for all optical measurement of elastic constants in NLCs.

In the experimental setup we used the polarizer to control the power of the beam. For a planar configuration it was used $\lambda / 2$ phase plate to control the polarization of the beam (in homeotropic texture polarization of the beam is not important). For measurements with an Nd:YAG laser a lens and a pinhole were used.

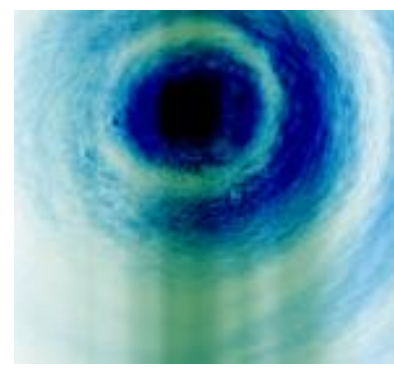

a)

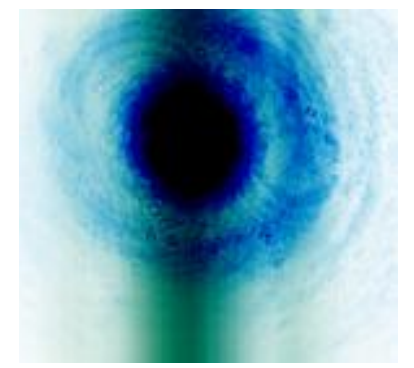

b)
Fig. 3. Pictures from experiment: a) 1110 in planar texture, $\mathrm{P}_{\mathrm{th}}=577 \mathrm{~mW}, \mathrm{~d}=45 \mu \mathrm{m}$; b) 1110 in hometropic texture, $\mathrm{P}_{\mathrm{th}}=363 \mathrm{~mW}$, $\mathrm{d}=35 \mu \mathrm{m}$.

A local change in the orientation of NLCs created by electric fields of electro-magnetic wave causes appearance rings which can be observed on the CCD camera (Fig. 3). Self-diffraction rings are observed because this field reorients directors and changes the phase of the beam passing through the NLCs. Elastic constants were measured and calculated for four different NLCs: 6CHBT (4-trans-4'-n-hexyl-cyclohexylisothiocyanatobenzene), 5CB (4-n-pentyl-4-cyanobiphenyl) and two mixtures 1110 and 1550 prepared by Prof. Dąbrowski's group from the Military University of Technology. The results of these measurements are presented in Table 1.

Table 1. Results of measurements of Frank's elastic constants from the experiment and results from the experiment with an electro-optical effect.

\begin{tabular}{|c|c|c|c|c|c|}
\hline $\mathbf{L C}$ & $\begin{array}{c}\mathrm{T} \\
{\left[{ }^{\circ} \mathrm{C}\right]}\end{array}$ & $\Delta \varepsilon$ & $\mathbf{K}_{\mathbf{2 2}}[\mathbf{p N}]$ & $\mathbf{K}_{\mathbf{3 3}}[\mathbf{p N}]$ & Data from: \\
\hline $6 \mathrm{CHBT}$ & - & - & 3.5 & 9.5 & {$[6]$} \\
\hline $5 \mathrm{CB}$ & - & - & - & 6.5 & {$[6]$} \\
\hline $6 \mathrm{CHBT}$ & 23 & 0.4794 & $3.5 \pm 0.7$ & $9.6 \pm 1.7$ & measurement \\
\hline $5 \mathrm{CB}$ & 21 & 0.6209 & - & $6.7 \pm 1.6$ & measurement \\
\hline 1550 & 23 & 0.1844 & $6.7 \pm 1.6$ & $19.3 \pm 2.3$ & measurement \\
\hline 1110 & 21 & 0.1359 & $8.0 \pm 0.9$ & $21.4 \pm 1.3$ & measurement \\
\hline
\end{tabular}

Cell thicknesses were $35 \div 52 \mu \mathrm{m}$. The threshold power for $6 \mathrm{CHBT}$ and $5 \mathrm{CB}$ in was the range $25 \div 56 \mathrm{~mW}$, for 1550 and 1110 these values were $130 \div 570 \mathrm{~mW}$. The elastic constant calculated from these values was compared with data from reference. The results can be also compared with measurements of spatial solitons in NLCs (nematicons). The nonlinear effect connected with 
reorientational nonlinearity requires power proportional to $\gamma_{i i}^{-1}$ where $\gamma_{i i}=K_{i i}(\Delta \varepsilon)^{-1}$, and $K_{\mathrm{ii}}(\mathrm{i}=1,2,3)$ is taken for the given configurations [7]. The creation of nematicons in chiral 1110 NLC requires $7 \div 8$ times larger power than for nematicons in 6CHBT [8]. This is in good agreement with our experimental results, where $\gamma_{22}^{(1110)} / \gamma_{22}^{(6 C H B T)}=7.5$. Similar agreement is obtained for nematicons in 1550 NLC, where this power is $5 \div 6$ times larger than in 6CHBT [9], and in our measurement $\gamma_{22}^{(1550)} / \gamma_{22}^{(6 C H B T)}=5.1$.

The threshold value [Eq. (3)] is calculated for a homogeneous field, which means that it is correct for a plane wave. Therefore it is important to use a relatively wide Gaussian beam to obtain the correct results. In our experiment we used beams with $\mathrm{w}_{0} \geq 10 \mu \mathrm{m}$. Another source of incorrectness is connected with precise calculation of an electric field in the center of the beam $\mathrm{E}_{0}$. Consequently, the beam shape and its size should be determined with high precision. In our experiment the value of the beam waist has been calculated using the divergence angle method.
Concluding, the measurement of Frank's elastic constants by using nonlinear effect seems to be a very good method giving results comparable to other methods and experiments in nonlinear optics. Additionally, in the all-optical method the threshold value of power for twist deformation (connected with $\mathrm{K}_{22}$ ) can be measured much more easily than in methods based on electro-optical effect.

This work was financially supported by the National Science Center.

\section{References}

[1] T. Wu, Phys. Rev. A, 331270 (1986).

[2] J. Kędzierski, Z. Raszewski, J. Rutkowska, T. Opara, J. Zieliński, J. Żmija, R. Dąbrowski, Mol. Cryst. Liq. Cryst. 249, 199 (1994).

[3] W.K. Bajdecki, M.A. Karpierz, Acta Phys. Pol. A 95, 793(1999).

[4] L. Calero, W.K. Bajdecki, R. Meucci, Opt. Comun. 168, 201 (1999)

[5] I.-Ch. Khoo,S. T. Wu, Optics and Nonlinear Optics of Liquid Crystals (Singapore, World Scientific Publ. 1993).

[6] Liquid crystals databas, LiqCryst 4.2, (C) 2002 Volkmar Vill.

[7] A. Piccardi, M. Trotta, M. Kwaśny, A. Alberucci, R. Asquini, M. Karpierz, A. d'Alessandro, G. Assanto, Appl Phys B 104, 805 (2011).

[8] Experiments done by U.A. Laudyn, M. Kwaśny and M.A. Karpierz

[9] U.A. Laudyn, K. Jaworowicz, M.A. Karpierz, Appl. Phys. Lett. 94, 091110 (2009); doi: 10.1063/1.3093489 